\title{
Modeling Characterization of the National Ignition Facility Focal Spot
}

\author{
W. H. Williams \\ J. M. Auerbach \\ M. A. Henesian \\ J. K. Lawson \\ J. T. Hunt \\ R. A. Sacks \\ C. C. Widmayer
}

This paper was prepared for and presented to the

SPIE International Symposium on

High-Power Lasers and Applications

San Jose, California

January 24-30, 1998

January 16, 1998

This is a preprint of a paper intended for publication in a journal or proceedings. Since changes may be made before publication, this preprint is made available with the understanding that it will not be cited or reproduced without the permission of the author. 


\section{DISCLAIMER}

This document was prepared as an account of work sponsored by an agency of the United States Government. Neither the United States Government nor the University of California nor any of their employees, makes any warranty, express or implied, or assumes any legal liability or responsibility for the accuracy, completeness, or usefulness of any information, apparatus, product, or process

disclosed, or represents that its use would not infringe privately owned rights. Reference herein to any specific commercial product, process, or service by trade name, trademark, manufacturer, or otherwise, does not necessarily constitute or imply its endorsement, recommendation, or favoring by the United States Government or the University of California. The views and opinions of authors expressed herein do not necessarily state or reflect those of the United States Government or the University of California, and shall not be used for advertising or product endorsement purposes. 


\title{
Modeling characterization of the National Ignition Facility focal spot
}

\author{
Wade H. Williams, Jerome M. Auerbach, Mark A. Henesian, Janice K. Lawson, \\ John T. Hunt, Richard A. Sacks, C. Clay Widmayer \\ Lawrence Livermore National Laboratory, Livermore, CA 94550
}

\begin{abstract}
The predicted focal spot size of the National Ignition Facility laser is parameterized against the finish quality of the optics in the system. Results are reported from simulations which include static optics aberrations, as well as pump-induced distortions, beam self-focusing, and the effect of an adaptive optic. The simulations do not include contributions from optics mounting errors, residual thermal noise in laser slabs from previous shots, air turbulence, a kinoform phase plate, or smoothing by spectral dispersion (SSD). Consequently, these results represent "first shot of the day", without-SSD, predictions.
\end{abstract}

Keywords: National Ignition Facility, NIF, focal spot simulations, high-power lasers

\section{BACKGROUND}

The National Ignition Facility is a MJ-class laser currently being built at Lawrence Livermore National Laboratory (LLNL). It will consist of 192 individual Nd-glass lasers, each beam being approximatly 35 x $35 \mathrm{~cm}$ square, and each providing 10 $\mathrm{kJ}$ light on target. Each beam is genereated by passing a low-energy injection laser pulse into a chain of optics consisting of laser amplifier slabs (pumped with Xe flashlamps), optical spatial filters, and other optics. The $1.053 \mu \mathrm{m}$ light $(1 \omega)$ in the main chain is frequency-converted to $0.351 \mu \mathrm{m}(3 \omega)$ using potassium diphosphate (KDP) crystals in the target chamber. A schematic of the laser is shown in Figure 1.

The machine has various performance requirements to meet its various missions. Primary among these mission is the ability to provide short $(\sim 1 \mathrm{~ns})$, high power $(\sim 700 \mathrm{TW}$ total $)$ pulses in as small a focal spot as possible. As part of the continuing design work on NIF, this study was initiated to consider the size of the focal spot as a function of the quality of the optics used in the machine.

The focal spot is determined by the spatial variations in phase and intensity of the beam as it enters the final focus lens. In a well-relayed system, with little aperture-clipping of the beam, intensity modulations arise largely from phase modulations which have diffracted into amplitude noise. Consequently, the focal spot in NIF is dictated, almost exclusively, by phase noise added to the beam as it propagates through the chain. There are a number of contributors to this noise (see Table 1), several of which are addressed in this modeling. A discussion of the those included in the model is given below.

\section{Optics Quality}

In brief, the phase noise added to the beam by an optic can be characterized by the Power Spectral Density (PSD) of the phase, as provided, for example, by an interferrogram of the optic. For specification and modeling purposes, this PSD is broken into several pieces which have qualitatively different effects on the near or far-field beam. An example is shown in Figure 2. This figure is a combination of specification and measurement.

In the low-frequency portion of the spectrum $(0-\sim 20 \mu \mathrm{rad})$ the rms gradient of the phase is used to describe the optic aberrations. This formalism assumes an approximately Gaussian distribution of the phase gradients added to the beam as it passes through an optic. Numerical realizations of this approach were accomplished by convolving a Gaussian nearfield phase array with an array of random numbers from - 1 to 1 . This "random Gaussian phase screen" was then scaled to give the desired rms gradient. Figure 2 was constructed assuming a $75 \AA$ ^/cm rms phase gradient. This portion of the spectrum almost completely defines the central portion of the focal spot. Values of 50,75 , and $90 \AA / \mathrm{cm}$ were used in this study for the rms gradient. The middle value is near to the optic-average for parts on Beamlet (the NIF prototype laser at LLNL.) 
Table 1. Major contributors to focal spot.

\begin{tabular}{|c|c|c|}
\hline Contributor & $\begin{array}{l}\text { Included in } \\
\text { Current } \\
\text { Modeling? }\end{array}$ & Comments \\
\hline Optics quality & yes & $\begin{array}{l}\text { paramaterized by rms phase gradient at low spatial } \\
\text { frequency, and scaled Power Spectral Density } \\
\text { measurements at higher frequencies }\end{array}$ \\
\hline Pump-induced distortions & yes & as predicted by stress-optics code calculations \\
\hline Adaptive optic & yes & numberical adaptive optic models \\
\hline Non-innear B-integral) effects & yes & includes whole-beam self-focusing and beam break-up \\
\hline Air turbulence & no & $\begin{array}{l}\text { this is "first shot of the day" modeling, so no turbulence } \\
\text { from previous shots; "cold cavity" air turbulence is } \\
\text { assumed small }\end{array}$ \\
\hline $\begin{array}{l}\text { Residual thermal stresses in } \\
\text { laser glass from previous shots }\end{array}$ & no & $\begin{array}{l}\text { this is "first shot of the day" modeling, so no residual } \\
\text { thermal stress in slabs from previous shots }\end{array}$ \\
\hline $\begin{array}{l}\text { Mounting/gravity sag effects } \\
\text { on optics }\end{array}$ & no & assumed corrected by adaptive optic \\
\hline $\begin{array}{l}\text { Smoothing by Spectral } \\
\text { Dispersion }\end{array}$ & no & not applicable for the short pulse mission \\
\hline
\end{tabular}

This formalism also requires a near-field scale length for the aberrations. The rms gradient fully specifies the uncorrected focal spot, independent of this scale length. With the inclusion of an adaptive optic, however, which partailly corrects the aberrations, the scale length becomes important: longer scale lengths are more readily corrected by the adaptive optic (with its $8 \mathrm{~cm}$ spacing between actuators) than shorter scale lengths. For this modeling, a scale length based on measured parts was chosen. Anticipated scale lengths for NIF components are not well-determined at this point.

The second portion of the spectrum ( 20-100 $\mu \mathrm{rad})$ is the lower-frequency portion of what we term the "waviness regime" (referred to as "waviness-1" for this study). For this work it is parameterized by scaling measurements made on real parts, the dominant contributor being one particular Beamlet laser slab. In results given here, the PSD of this optic was used with magnitude as measured, or cut in half (which is a sqrt(2) multiplier on the phase). This portion of the spectrum contributes to the wings of the $1 \omega$ focal spot, as well as to near-field modulation of the beam. It also contributes source terms which can grow significantly on frequency conversion, causing a significant enlarging of the spot size from $1 \omega$ to $3 \omega$ at high powers.

The third portion of the spectrum (100-1000 $\mu \mathrm{rad})$ is the upper portion of the waviness specification (referred to as "waviness2 " in this study). Only a portion of this regime was accessed in these calculations, as we had an upper limit of $539 \mu$ rad due to the grid resolution used in simulations $(512 \times 512$ over $50 \times 50 \mathrm{~cm})$. This portion of the spectrum contributes negligibly to the focal spot size (except at very high powers). It does, however, contribute to near-field beam modulation and ablation of the edges of the pinholes in the spatial filters. The damage of optics by the high-fluence portions of the laser beam, which is driven by beam modulation, is included in the modeling, and sets the operating limit of the machine. Pinhole ablation is not currently modeled. For this study, measured PSD values were used from several measurements on real parts. They were not varied for this study since modeling of propagation of small patches of a beam is more suitable to studying these highfrequency contributors.

These three portions of the spectrum, their dominant effects, and the values used in this study are summarized in Table 2. In general, most of the rms gradient portion of the spectrum, and part of the waviness-1 regime, can be (partially) corrected by the adaptive optic. The waviness-2 regime is outside the range of correction.

Table 2. Three PSD regimes as used in modeling.

\begin{tabular}{|c|c|c|c|}
\hline Title & $\begin{array}{l}\text { Frequency } \\
\text { range }(\mu \mathrm{rad})\end{array}$ & Values used in study & Dominant Effect on beam \\
\hline rms gradient & $0-\sim 20$ & $50,75,90 \AA / \mathrm{cm}$ & main focal spot size \\
\hline waviness-1 & $\sim 20-100$ & $\begin{array}{l}\text { nominal*, } \\
(\text { nominal*)/2 }\end{array}$ & wings of focal spot; near-field modulation \\
\hline waviness-2 & $100-539$ & nominal* & near-field modulation; pinhole blow-off \\
\hline
\end{tabular}

* nominal as measured on specific, representative parts 


\section{Pump-Induced Distortions}

Pump-induced distortions arise as the laser slabs are irradiated by the flashlamps. They experience thermal stresses, temperature gradients, and bending modes from these gradients which result in a difference in optical path length through different portions of the slab. This is equivalent to phase noise being added to the beam as it passes through the slab.

The pump-induced distortion for this modeling was calculated using a set of LLNL stress-optics codes, including NIKE3D. It starts with the pumping profile on the slabs, as calculated from a ray trace code. The pump-induced phase noise at the time of laser pulse extraction for a representative slab is shown in Figures 3. Note that this is a long-wavelength aberration which is well-corrected by the adaptive optic.

\section{Adaptive Optic}

The adaptive optic serves to correct many of the long-wavelength aberrations on the beam. For this study, our adaptive optic model assumes Gaussian influence functions. In use, it adjusts each actuator to minimize the intensity-weighted rms phase across the beam. The influence function spacings are shown in Figure 4. The mirror has a residual error specification. This was not included in the modeling, making the results probably optimistic. In modeling, the correction was done at the input to the frequency converter. This is approximately correct. The diagnostic pick-off for the adaptive optic in NIF is just after the output lens of the transport spatial filter (see Figure 1), so the transport mirrors will not actually be corrected. This is a small effect, given our current models for the mirrors. Also, the correction will be done further up in the chain, at mirror 1. Simulations have shown, however, that the long-wavelength corrections provided by the adaptive optic remain as phase over the propagation distances in NIF, so they can be placed anywhere in the chain, to good approximation.

\section{B-Integral Effects}

The intensity-dependent, non-linear index of refraction of materials in the laser contributes to several focal spot effects, including whole-beam self-focusing, beam modulation/breakup, and spot size flaring on frequency conversion.

The relevant quantity for characterization in numerical studies is the "B integral", which can be thought of as the phase pushback in intense regions of the beam. It is defined as

$$
B=\frac{2 \pi}{\lambda} \int I(z) \gamma(z) d z
$$

where $\gamma$ defines the non-linear index of refraction from

$$
n=n_{0}+\gamma I
$$

and $I$ is the beam intensityand $\lambda$ is the wavelength. The integral is over any propagation distance of interest for the beam. The B integral for the entire chain (from beam injection to the input of the frequency conversion crystals) is termed " $\Sigma \mathrm{B} "$ in these results. The $\mathrm{B}$ integral between sequential spatial filter pinholes is termed " $\Delta \mathrm{B}$ ".

\section{Whole-Beam Self-Focusing}

The spatial profile of each NIF beam will be square, with an approximately flat-intensity center region of $33 \mathrm{x} 33 \mathrm{~cm}$, then tapering to zero intensity at $37 \times 37 \mathrm{~cm}$. Whole-beam self-focussing is an effect in this tapering portion of the beam, where the non-linear index of refraction causes a phase push-back proportional to this intensity profile, with the net effect that the edges of the beam are focused inward. With the NIF square beam, this is seen in the focal spot as streaks in the $\mathrm{x}$ and $\mathrm{y}$ directions. An example of a high-power calculated spot is shown in Figure 5, with the whole-beam effect noted. The spatial frequencies in the edge of the beam are within the bandpass of the spatial filter pinholes, so this effect is proportional to $\Sigma \mathrm{B}$ rather than $\Delta \mathrm{B}$.

\section{Beam Modulation/Breakup}

The non-linear index of refraction also causes growth of small-scale phase and intensity ripples ${ }^{1,2}$ The portion of this growth which is not clipped by pinholes, or which is after the last pinhole, contributes to an increase in the focal spot size with increasing output power. This has a relatively minor effect on $1 \omega$ spot size, compared to the whole-beam effect, but can contribute to the $3 \omega$ spot size at high power, as discussed in the next section. The beam modulation resulting from this growth, however, causes the collateral effect of high local fluences which cause damage to the optics. Indeed, the output power of the laser is limited by this near-field modulation and the resulting peak $3 \omega$ fluence exiting the frequency converter. 


\section{Spot Size Flaring on Frequency Conversion}

The dominant frequency conversion effect on spot size is mode-mixing of low-frequency components of the field, causing an enlargement of the main spot size. Mode mixing is a result of the non-linear processes in frequency conversion as sum and difference components arise from the original frequency components in the $1 \omega$ field. The sum components increase the size of the far field. This is largely independent of beam power. In the results that follow (cf. Figure 6) the $3 \omega$ spot size is seen to be a few $\mu$ rad larger than the $1 \omega$ spot from 0 to $4 \operatorname{rad} \Sigma B$, due to this effect.

Separately, the frequency conversion process also increases the amplitude of high-spatial frequency phase noise outside of the central region of the spot by up to a factor of nine ${ }^{3}$. If the amplitudes of these noise terms, as they arrive from the $1 \omega$ portion of the laser, are of sufficient magnitude, then one will see a noticeable difference in the spot size from $1 \omega$ to $3 \omega$. The magnitude of these sources is dictated both by the original sources (the optics specifications), and the $\Sigma \mathrm{B}$ growth in the $1 \omega$ laser. This increase in spot size, however, is in the wings of the spot. An example is shown in Figure 5, where highfrequency noise is visible in the $3 \omega$ spot, but much less in the $1 \omega$ spot (except for the whole-beam self-focusing noise). It only begins to effect the $80 \%$ spot size at relatively high powers where these wings contain a significant fraction of the beam energy (cf. Figure 6 where the $3 \omega$ spot size diverges dramatically from the $1 \omega$ spot at $\Sigma$ B $>4 \mathrm{rad}$ ).

\section{NUMERICAL MODEL}

This modeling utilized a 512 × 512 point, 50 x $50 \mathrm{~cm}$ grid with three time slices $(0.01, .98$, $0.01 \mathrm{~ns})$. It was done with LLNL propagation and frequency conversion codes (PROP92 and THG403). Phase noise as described above was placed on each optic, and pump-induced distortions on the amplifier slabs. The component spacings were taken from the current NIF optical layout. All results here were done with a $1 \mathrm{~ns}$, temporally flat output pulse at varying powers. Spatially-dependent gain files, approximately corresponding to current estimates for NIF were used. The pinhole cutoff angles were $\pm 100 \mu$ rad. The injection pulse from the front end had a flat phase. This is due to currently incomplete specification of the front end, making modeling uncertain. Measurements on Beamlet, however, indicate this assumption is not unreasonable

\section{RESULTS}

Calculations were done for four combinations of optics specifications:

\begin{tabular}{|c|c|c|}
\hline Case & $\begin{array}{l}\text { rms Gradient } \\
(\AA / \mathrm{cm})\end{array}$ & $\begin{array}{l}\text { Waviness-1 (scalar } \\
\text { multiple of "nominal") }\end{array}$ \\
\hline 1 & 90 & 1 \\
\hline 2 & 75 & 1 \\
\hline 3 & 75 & 0.5 \\
\hline 4 & 50 & 0.5 \\
\hline
\end{tabular}

A set of eight output powers were run for each case. These were parameterized as the $1 \omega \Sigma \mathrm{B}$ from the injection lens to the input of the converter, ranging from 0 to 5.5 radians.

Results are shown in Figures 6 through 9. Figure 6 shows the output power at the target plane, the damage fraction at the converter, and the $80 \% 1 \omega$ and $3 \omega$ spot sizes. The target plane energy is independent of the optics specifications, within the limit that only a small fraction of the beam energy is dumped on spatial filter pinholes (valid for these calculations). The damage converter fraction is the ratio between the peak fluence anywhere in the beam and the fluence at which the component is expected to damage. For NIF, the damage-limiting component is the tripler crystal of the converter at $3 \omega$. At low fluences, the damage fraction is proportional to output power. At higher fluences, however, non-linear noise growth makes the peak fluence superlinear in output power $(\Sigma \mathrm{B})$. This can be seen in Figure 6 . The $80 \%$ spot size is that half-angle which contains $80 \%$ of the spot energy.

It can be seen in Figure 6 that for all cases, the tripler crystal damage fluence exceeds the limit at a $\Sigma B$ of $\sim 3.5$ radians. We set this as the functional operating limit of the laser. From the same figure, at this operating point, the output energy of the laser is $\sim 700 \mathrm{~kJ}$. The $1 \omega 80 \%$ spot size ranges from 12 to $18 \mu \mathrm{rad}$ at the operating point for the four cases. The $3 \omega$ spot size is 14 to $22 \mu \mathrm{rad}$.

The effect of frequency conversion on spot size is also illustrated in this figure. Comparing the $1 \omega$ and $3 \omega$ spot sizes one can see that the $3 \omega$ spot is a few $\mu$ rad larger at all output powers up to $\Sigma B \sim 3.5$. This is due to the low-frequency mode mixing discussed above. The flaring of the spot above $\Sigma \mathrm{B} \sim 3.5$ is due to the large growth of high-frequency terms on conversion also discussed above. At this $\Sigma \mathrm{B}$ their magnitude in the $1 \omega$ laser have grown to sufficient magnitude that on conversion they account for a significant portion of the spot energy. 
Figure 7 shows the $1 \omega$ and $3 \omega$ fluence contrast ratio. The contrast ratio is defined as an rms variation in fluence across the beam from the mean, and is a measure of the modulation of the near-field fluence. Note the dip in both these figures at $\Sigma \mathrm{B}$ 1-3 radians. Several effects contribute to this behavior. The initial $1 \omega$ decrease is (probably) due to gain saturation effects, followed by the increase due to non-linear growth of high-frequency terms. At $3 \omega$, the initial decrease is thought to be primarily due to the converter response. The plane-wave conversion curve for the NIF design is shown in Figure 8 . At low $1 \omega$ intensities the curve has a larger slope than at higher intensities. Consequently, at low intensities the near-field modulation is magnified as the peaks in the beam convert better than the valleys. As the intensity increases, however, the converter curve flattens, decreasing the $3 \omega$ contrast until it approaches the $1 \omega$ contrast. The contrast begins to increase again, however, as the $1 \omega$ contrast is increasing due to non-linear growth in the $1 \omega$ chain. In addition, however, this $1 \omega$ noise which causes the modulation can be magnified by as much as a factor of nine on conversion (as discussed above), increasing the $3 \omega$ contrast above that at $1 \omega$, as seen in the figure.

A reasonable rule-of-thumb limit for the contrast ratio is $\sim 10 \%$, giving a peak-to-mean modulation of approximately $1.3: 1$ $(3 \sigma$, where $\sigma$ is the contrast ratio $=0.1)$. From this figure, one can see that this limit is exceeded in the $3 \omega$ beam at approximately $\Sigma \mathrm{B}=3$ to 4 . This is coincidentally consistent with the limit set by the damage results in Figure 6 .

Figure 7 also shows the fraction of energy outside a $33 \mu \mathrm{rad}$ circle. At the operating point this ranges from 4 to $6 \%$ at $1 \omega$ and 8 to $10 \%$ at $3 \omega$.

Figure 9 shows the $50 \%$ and $95 \%$ spot sizes. At the operating point, the $50 \%$ spot size ranges from 2 to $10 \mu$ rad at $1 \omega$, and 8 to $12 \mu \mathrm{rad}$ at $3 \omega$. The very small size for the $50 \AA / \mathrm{cm}$ case $(2 \mu \mathrm{rad})$ is thought to be very sensitive to uncertainties in the adaptive optic model. More importantly, it is at the limit of the calculational grid $\left(\theta_{\min }=2 \mu \mathrm{rad}, \Delta \theta=2 \mu \mathrm{rad}\right)$, so is unreliable. The $95 \%$ spot size radius ranges from 30 to $37 \mu \mathrm{rad} 1 \omega$, and 48 to $50 \mathrm{mrad} 3 \omega$.

\section{CONCLUSIONS}

Spot size calculations have been done for a NIF $1 \mathrm{~ns}$, high power pulse. The model includes aberrations from the passive optics, pump-induced distortions, self-focusing effects, and the adaptive optic. Aberrations from residual thermal effects, air turbulence, SSD, and mounting effects have not yet been included.

For this temporally flat $1 \mathrm{~ns}$ pulse, the operating limit of the machine is approximately $700 \mathrm{~kJ}(700 \mathrm{TW})$. Near this point we simultaneously find the peak fluence in the tripler meeting the damage threshold, the $3 \omega$ contrast ratio exceeding $10 \%$ (related to the peak fluence), and the spot size beginning to grow non-linearly on frequency conversion.

\section{ACKNOWLEDGEMENTS}

The authors would like to acknowledge the contributions of K. Jancaitis, J. Lawson, K. Manes, C. Orth, M. Rotter, and J. Trenholme in this work. This work was performed under the auspices of DOE by LLNL under contract W-7405-Eng-48.

\section{REFERENECES}

1. V. I. Bespalov, V. I. Talanov, "Filamentary structure of light beams in non-linear liquids", JETP Lett., 3, 307 (1966).

2. J. T. Hunt, K. R. Manes, P. A. Renard, "Hot images from obscurations", Appl. Opt., 32 (30), 5973 (1993).

3. J. M. Auerbach, D. Eimerl, D. Milam, P. W. Milonni, Appl. Optics, 36(3), 1997. 


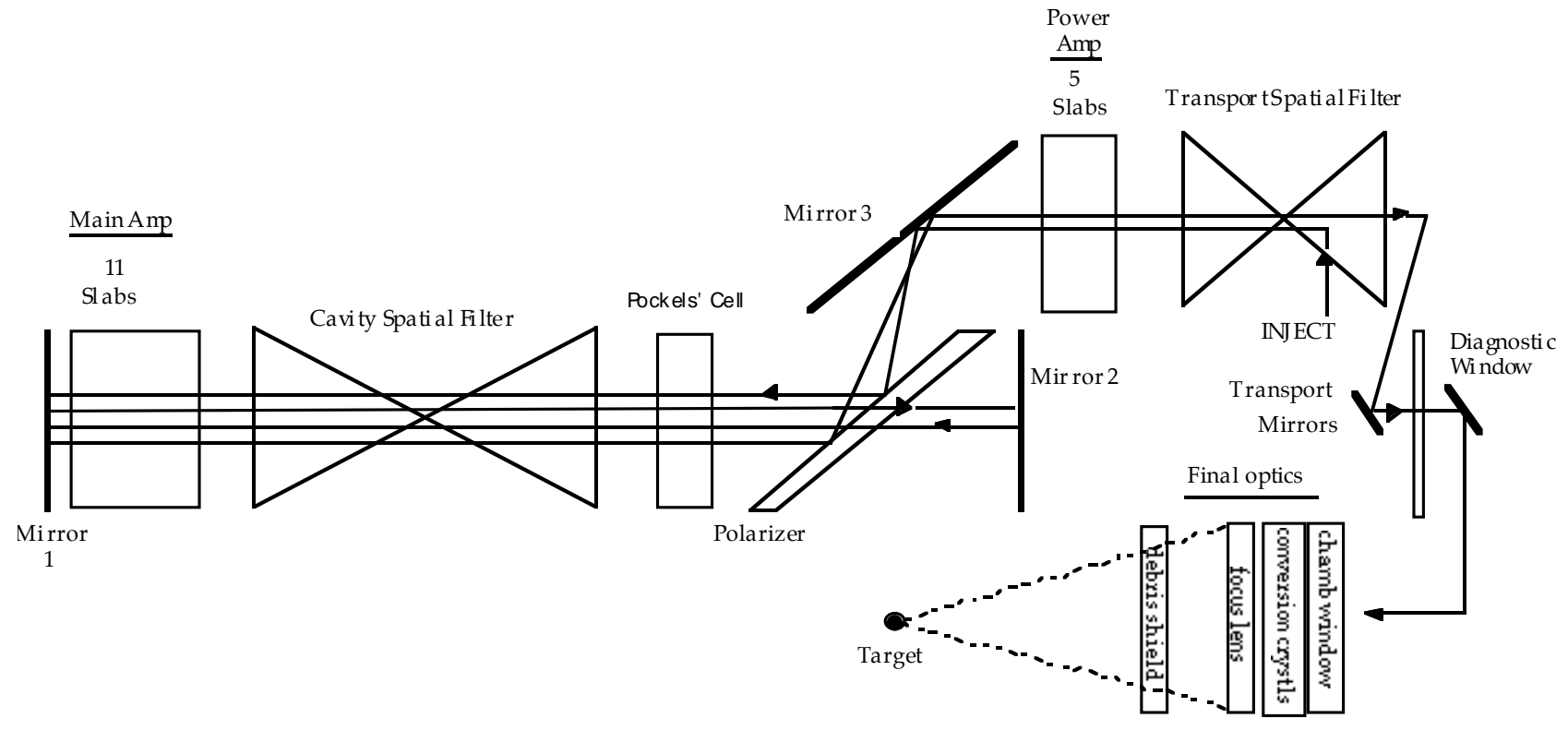

Figure 1. Scehmatic of layout of a single NIF beamline (not to scale).

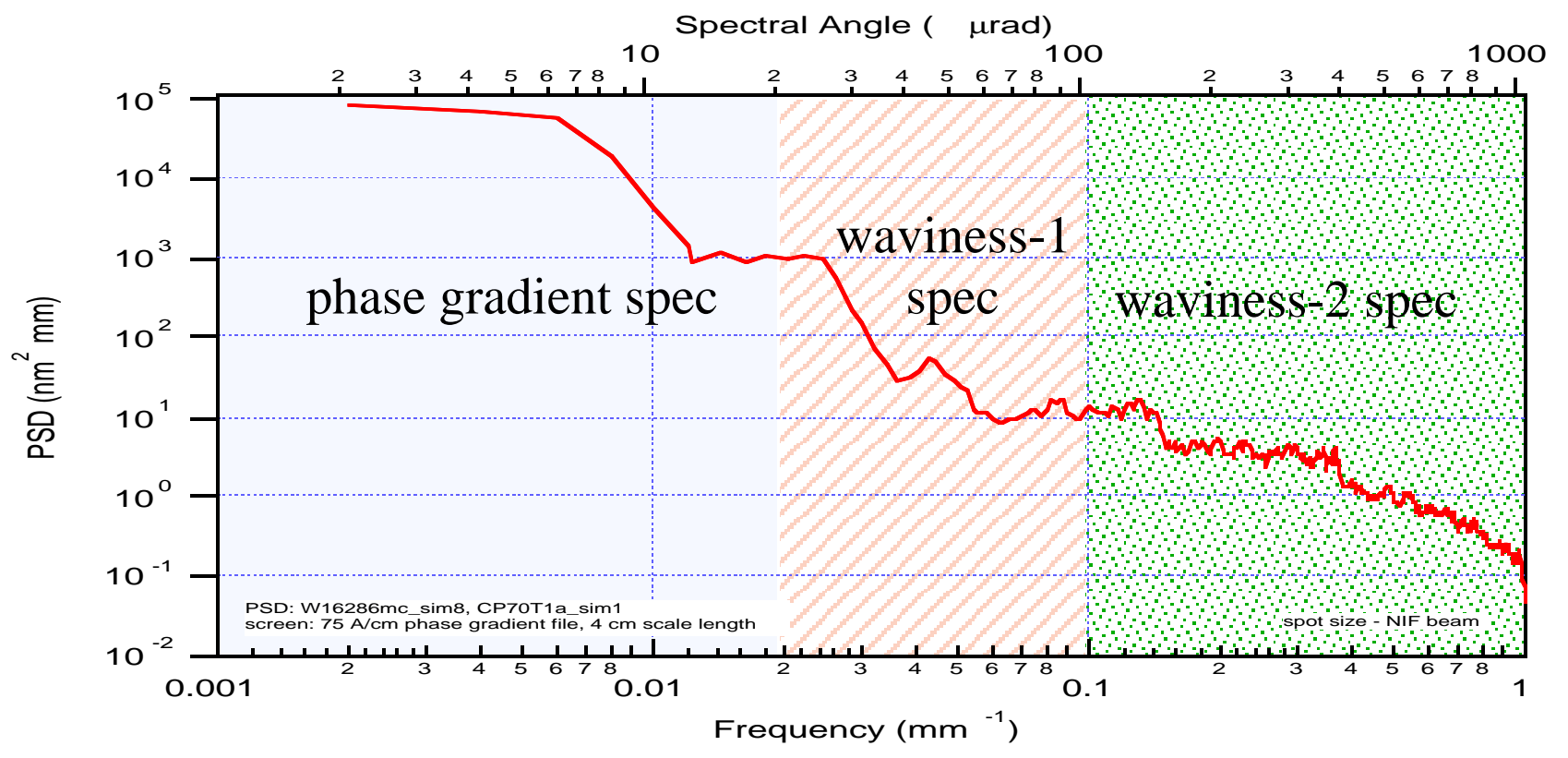

Figure 2. The phase noise added by an optic is described by its Power Spectral Density (PSD) curve, and for an optic is broken into three pieces for calculational convenience. The low-frequency portion is described by the rms gradient specification, and the other two by scaled PSD measurements. This curve corresponds to a laser slab with rms gradient of 75 $\AA / \mathrm{cm}$, and "nominal" (i.e. as measured on a representative part) PSD at higher frequencies. 

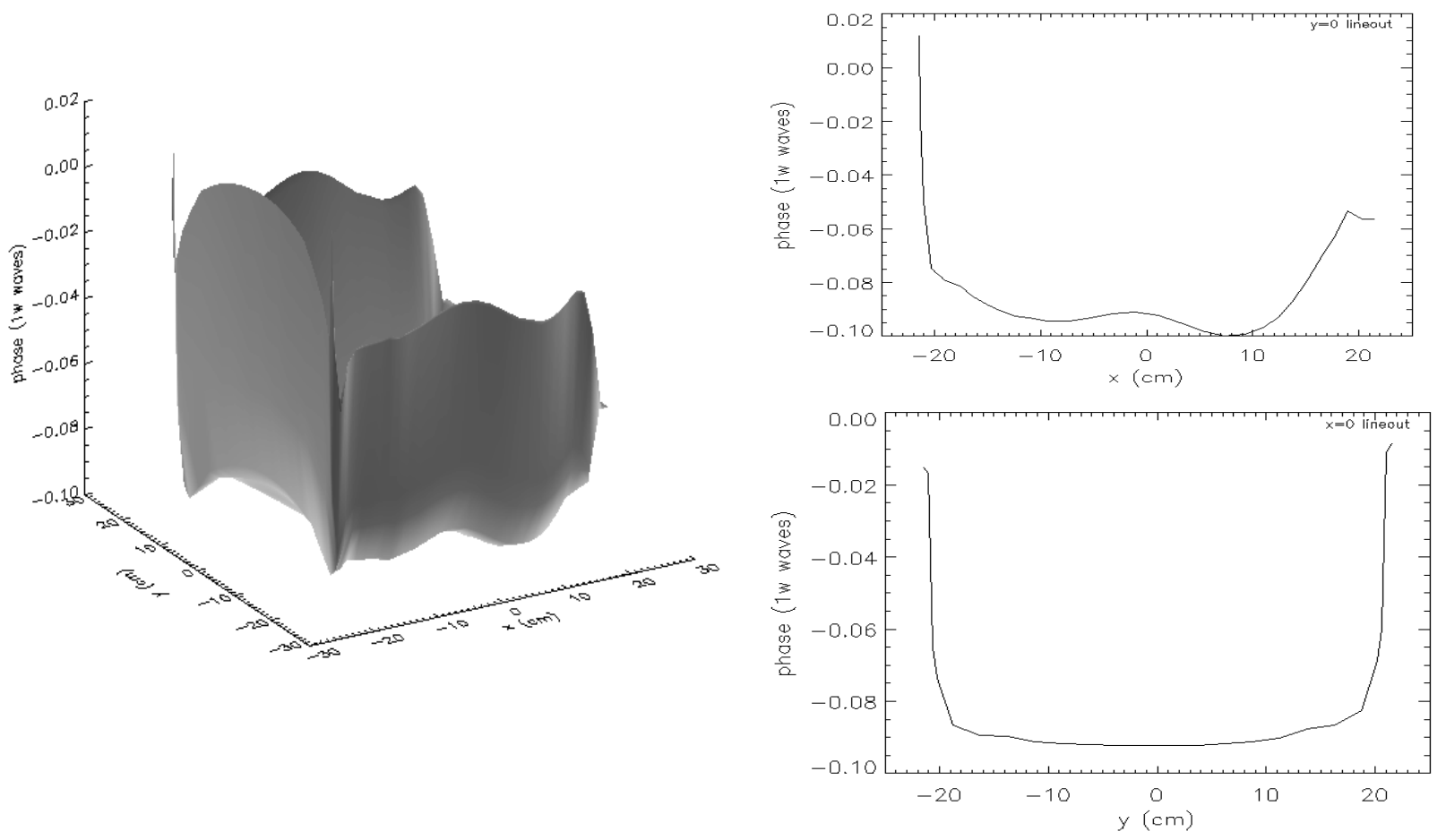

Figure 3. Phase noise added by pump-induced distortion in a laser slabs, as calculated from stress-optics codes. The large distortion at the edges is due to edge-clad heating.

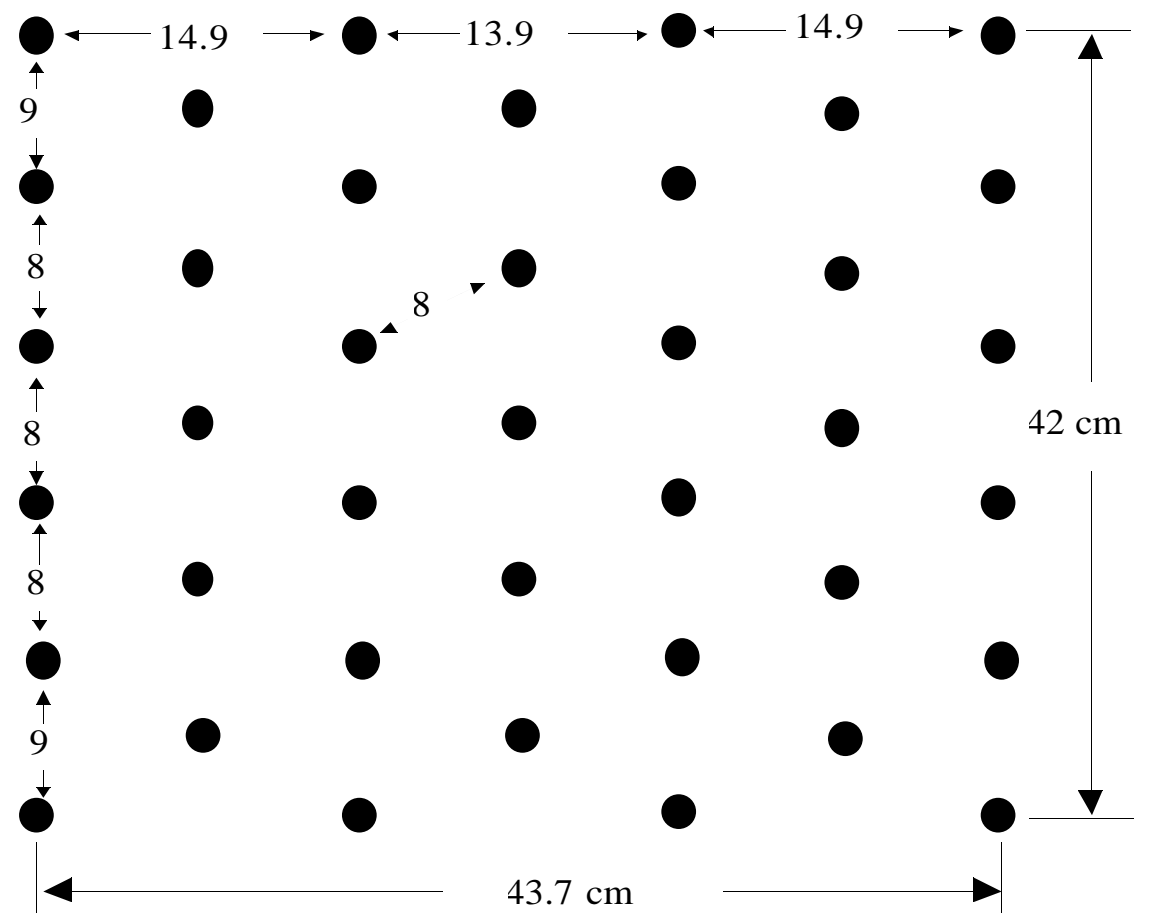

Figure 4. Location of influence function centers for adaptive optic model used in calculations. The influence functions were assumed to be Gaussian, with 1/e widths of $7.323 \mathrm{~cm}$. 


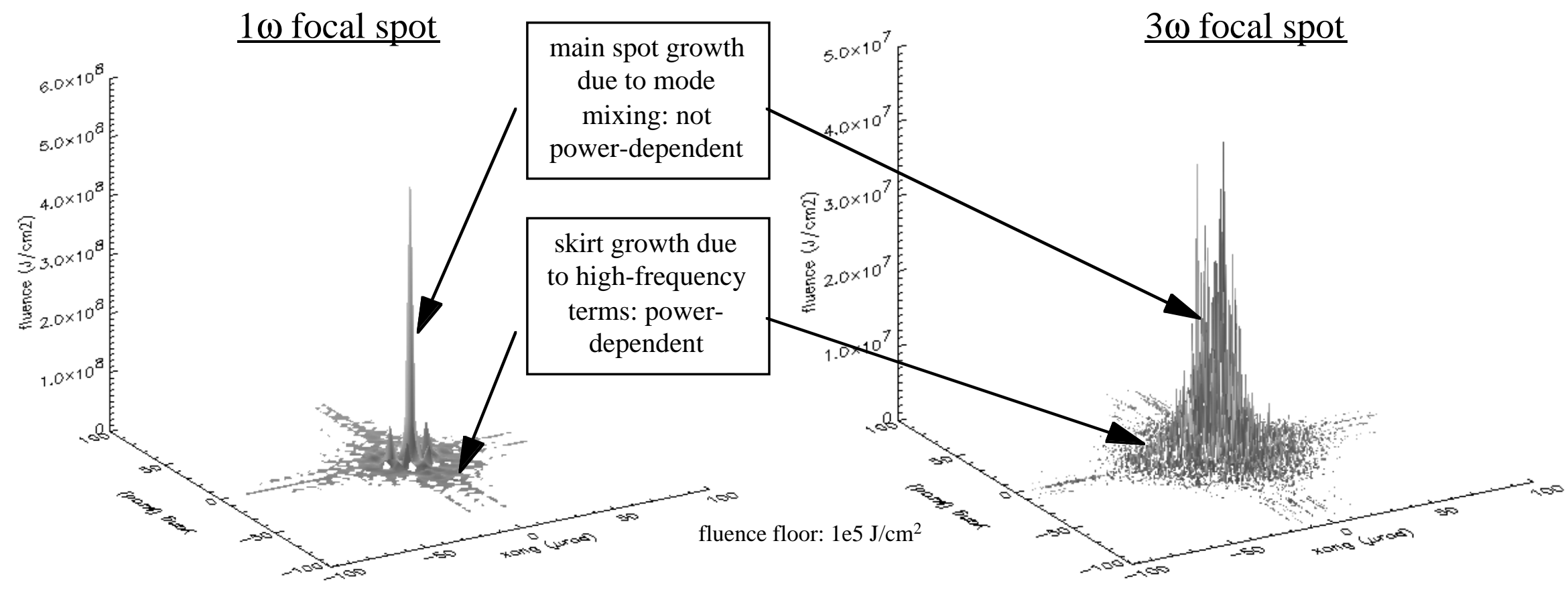

Figure 5. Comparison of $1 \omega$ and $3 \omega$ spot sizes at $\Sigma \mathrm{B}=5$ for the $90 \AA / \mathrm{cm}$ case. The increase in the size of the central portion of the spot is due to non-linear mode-mixing of low-frequency terms on tripling, and is approximately independent of beam power. The high-frequency noise around the base of the $3 \omega$ spot is due to growth of the $1 \omega$ source terms (up to a factor of 9) on conversion. The magnitude of these source terms is dependent on beam power (i.e. $\Sigma \mathrm{B}$ ). In this case, they are of sufficient magnitude after tripling that the $80 \%$ spot size is significantly effected. The characteristic X pattern is due to whole-beam self-focusing. 

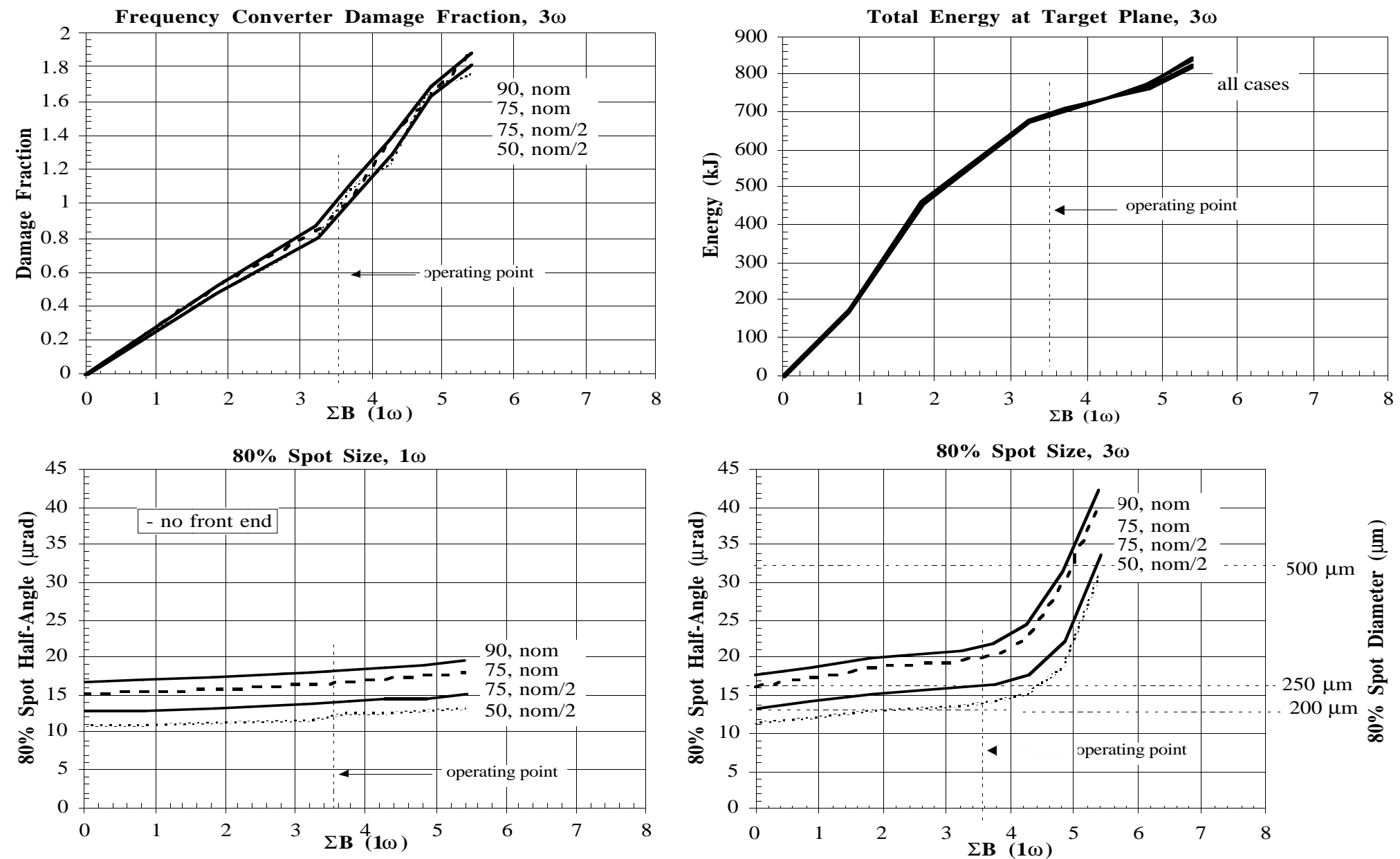

Figure 6. Converter $3 \omega$ damage fraction, total energy on target, and $80 \%$ spot sizes. The $\Sigma \mathrm{B}$ at which a unity converter damage fraction is exceeded sets the operating point for the laser at $\Sigma \mathrm{B}=3.5$ radians. This corresponds to $\sim 700 \mathrm{~kJ}$ on target, with $80 \%$ spot sizes from 14 to $22 \mu$ rad for the four cases considered. 

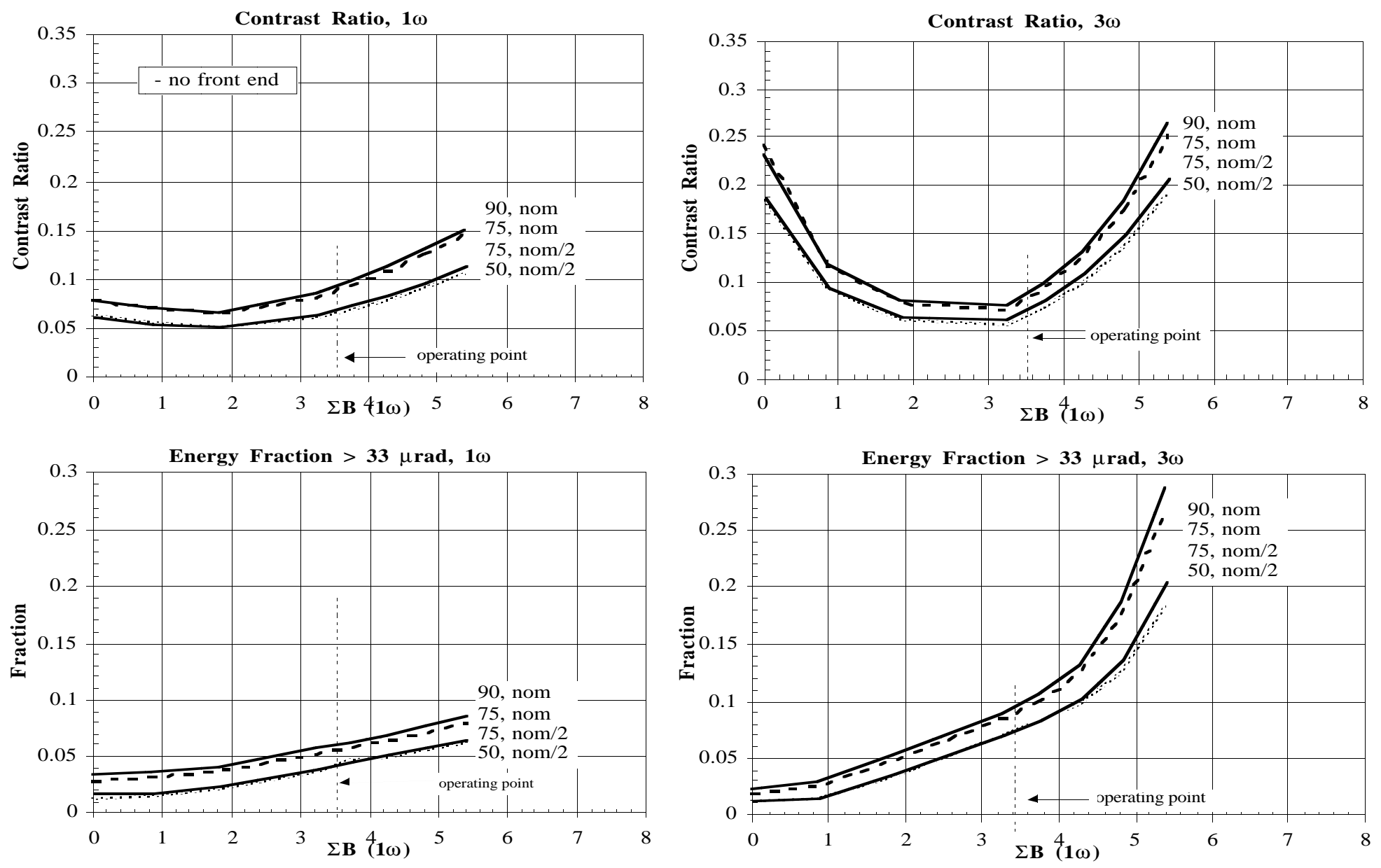

Figure 7. Contrast ratio and fraction of energy at angles $>33 \mu \mathrm{rad}$. See text for an explanation of the concave shapes in the $1 \omega$ and $3 \omega$ contrast ratios. A reasonable limit for the contrast ratio is $10 \%$. 


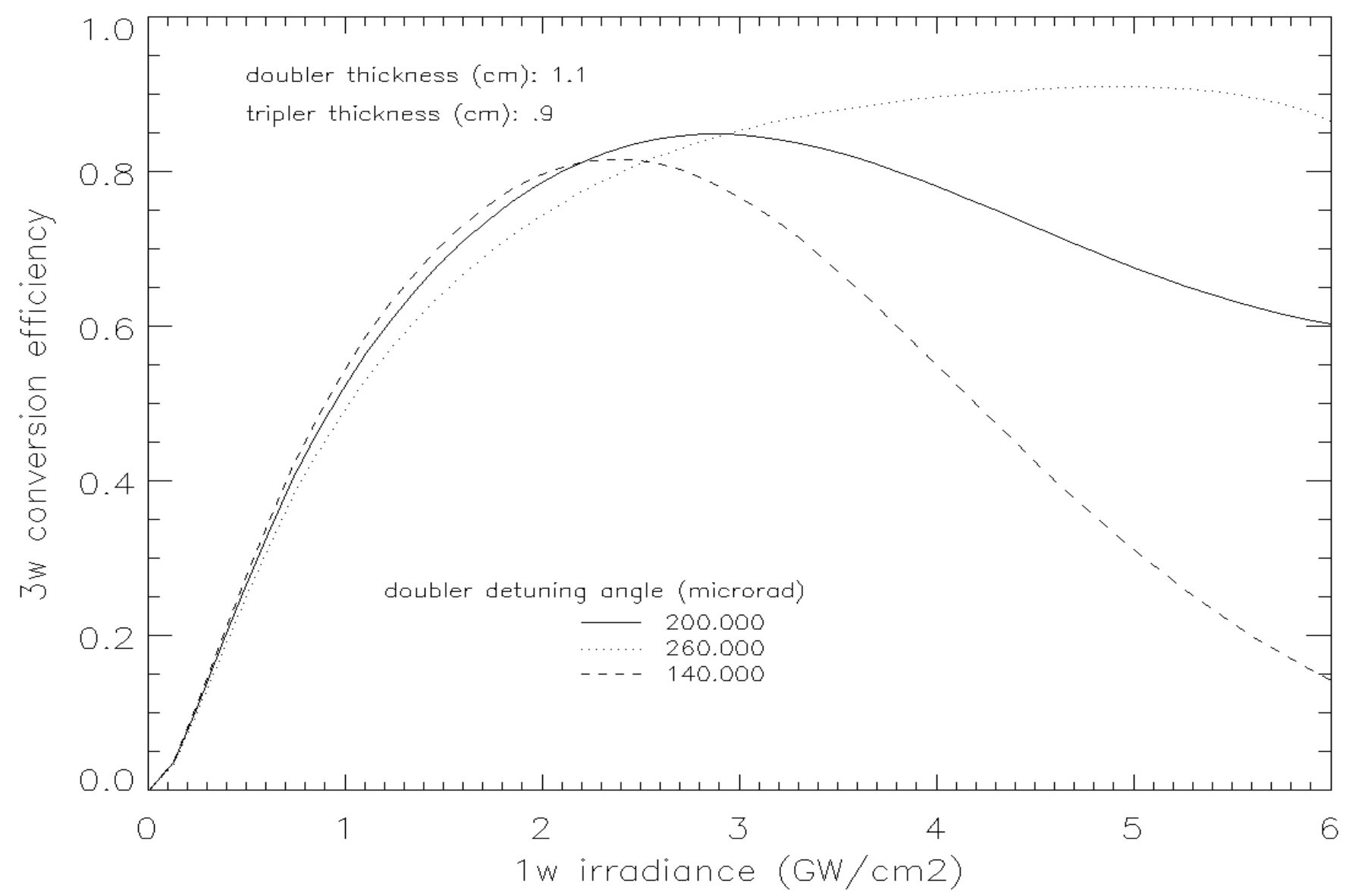

Figure 8. Plane wave frequency converter performance. The steep slopes at low intensities (e.g. $<1 \mathrm{GW} / \mathrm{cm}^{2}$ ) cause the $3 \omega$ contrast ratio to be larger than that at $1 \omega$. 

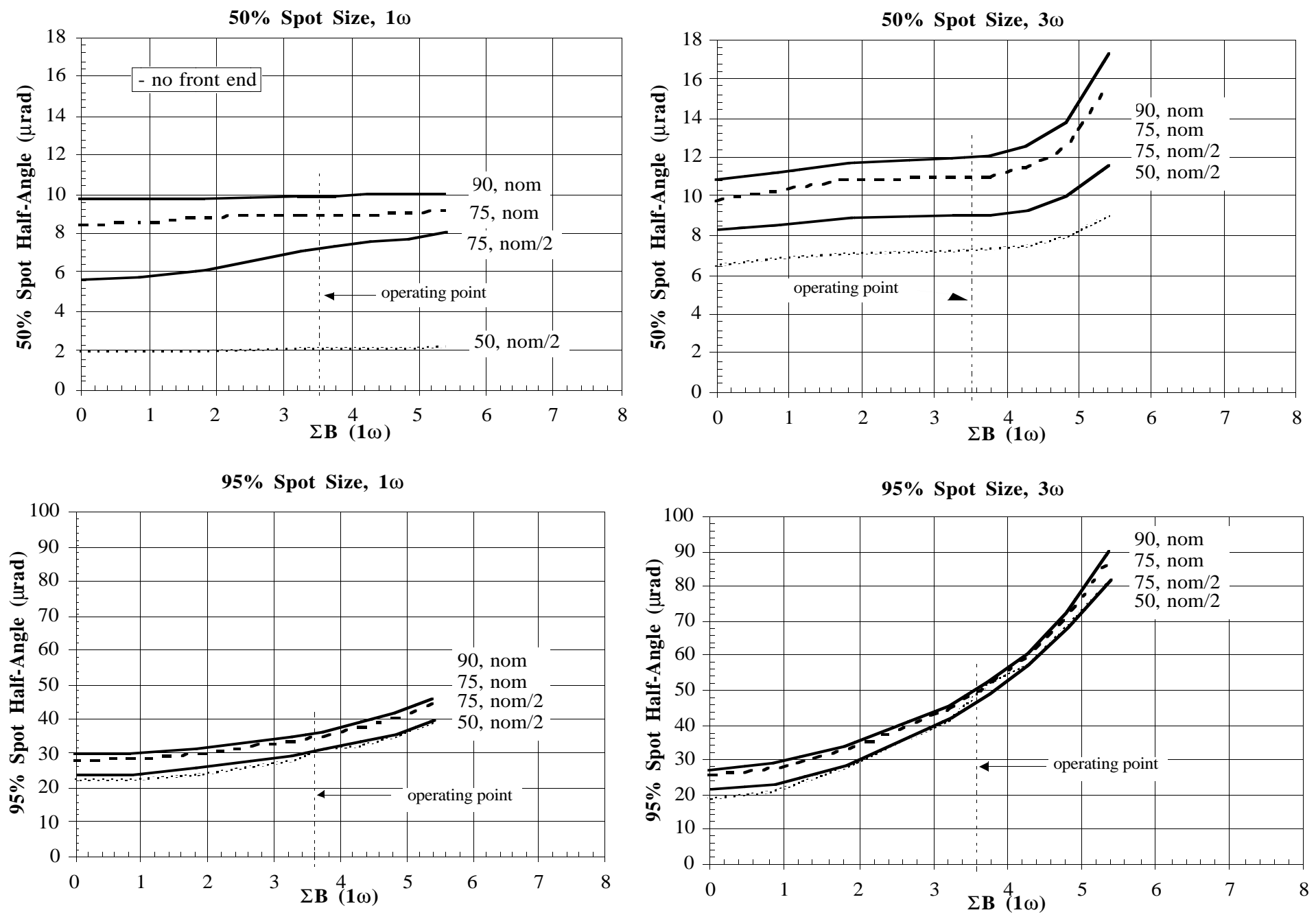

Figure9. $50 \%$ and $95 \%$ spot sizes. The $2 \mu \mathrm{rad} 50 \%$ spot sizes for the $50 \AA / \mathrm{cm}$ case are invalid, due to the adaptive optic model and calculational gird used. 